\title{
Unfavorable outcome of a paranasal cosmetic prosthesis caused by dental implants: a case report
}

\author{
Hyunmi Jo, Yeonjin Oh, Bong-Jin Jeong, Junho Jung, Joo-Young Ohe \\ Department of Oral and Maxillofacial Surgery, Kyung Hee University School of Dentistry, Seoul, Korea
}

\begin{abstract}
This article presents an unfavorable outcome of a paranasal cosmetic prosthesis, Medpor, which is affected by dental implants. The paper provides treatment insight for oral and maxillofacial specialists. The patients had undergone mandibular set-back surgery combined with both paranasal augmentations using Medpor. In due course, dental implants were placed on the anterior maxilla a few years later. The patient suffered from persistent pain and purulent discharge, but no significant correlation was observed on the panorama and CBCT. Dental implants were inserted near the Medpor for a paranasal augmentation, affecting each other, resulting in clinical symptoms. Medpor, as a radiolucent material, was not visible on dental radiography. Its characteristics of a bony-like texture and color could mislead dentists to misjudge a bony structure clinically. Therefore, the dental implant fixtures were inserted near the Medpor. The Medpors on the affected area were infected, requiring it to be surgically removed. This suggests that both dental implants and Medpors failed. A comprehensive evaluation of patient history and strategic presurgical planning should be obtained to avoid an inappropriate consequence of grafting. (JOURNAL OF DENTAL IMPLANT RESEARCH 2020;39(3):29-33)
\end{abstract}

Key Words: Medpor, Dental implant, Complications

\section{INTRODUCTION}

An appropriate midfacial volume is related to having an impression of youthful and possessing an attractive profile. A deep nasolabial angle and flattened profile caused by the deficiency of the paranasal area may become an aesthetic complaint ${ }^{1,2)}$. Several surgical methods to improve midfacial contour could be suggested with advance Le fort I osteotomy, malar osteotomy or simple augmentation using various graft material ${ }^{3)}$. The highdensity porous polyethylene (Medpor, Porex Surgical Inc., GA, USA), the alloplastic reconstructive implant, has been widely used in the craniofacial reconstruction and aesthetic augmentation with many advantages such as its biocompatibility, suitable strength and intraoperatively easy manipulation ${ }^{4,5)}$. The characteristics of Medpor include a bony-like texture, white color and radiolucency on imaging. Medpor has been reported as an optimal prosthesis for craniofacial reconstruction with few complications except rare fracture, exposure, discomfort and infection $^{6,7)}$.

Meanwhile, dental implants, the first consideration for replacing partially missing teeth, is becoming one of the most popular treatment in dentistry. Dental implant success is related to a rehabilitation of function, esthetics as natural tooth as well as bone physiology leading to osseointegration ${ }^{8)}$. It requires a comprehensive evaluation of the available bone and the local anatomical environment. On the anterior maxilla, dental implant installation could be a challenge arising from limited alveolar bone width, thin, soft tissue and anatomical variations ${ }^{9,10)}$.

Here, an unusual complication combined with Medpor and dental implant on the anterior maxilla are introduced for the first time. The purpose of this article is to present a malpositioned dental implant fixture on the radiographically non-visible cosmetic prosthesis. Moreover, it

Received September 7, 2020, Revised September 20, 2020, Accepted September 21, 2020

(C) Journal of Dental Implant Research.

(i) This is an open access article distributed under the terms of the Creative Commons Attribution Non-Commercial License (http://creativecommons.org/licenses/by-nc/4.0) which permits unrestricted non-commercial use, distribution, and reproduction in any medium, provided the original work is properly cited.

Correspondence to: Joo-Young Ohe, Department of Oral and Maxillofacial Surgery, Kyung Hee University School of Dentistry, 26 Kyungheedae-ro, Dongdaemun-gu, Seoul 02447, Korea. Tel: +82-2-961-9360, Fax: +82-2-958-9344, E-mail: ojyoung81@khu.ac.kr 


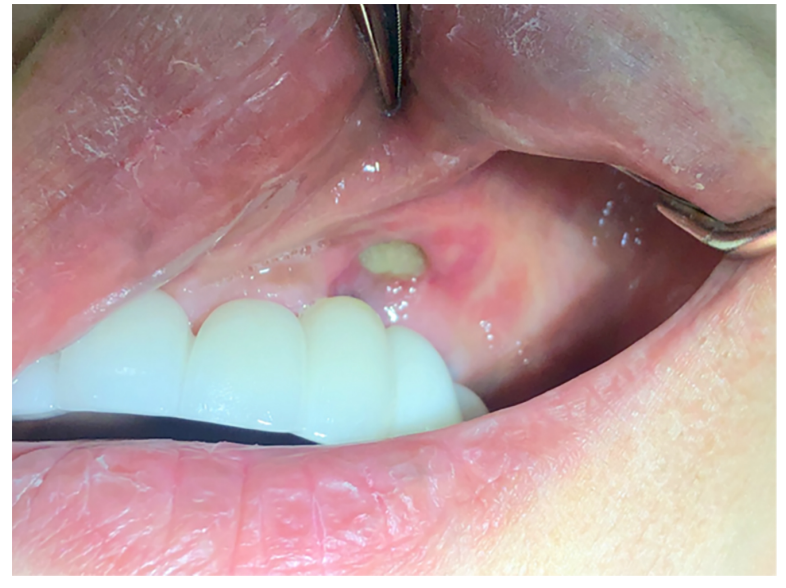

Fig. 1. On the first visit, a bony-like surface was exposed under \#23i prosthesis. Pus discharge was no more observed, however, mild swelling and redness around the lesion remained as well as soft tissue was lost.

suggests insight in to the treatment procedures for oral and maxillofacial specialist and emphasizes the importance of pre-operative patient evaluation.

\section{CASE REPORT}

A 34-year-old female who had no specific medical problem was referred from local clinic to the department of Oral and Maxillofacial surgery at Kyung Hee University Dental Hospital on December 03 2019. The chief complaint was the untreatable bony exposure and purulent discharge on left maxillary mucosa. The doctor who sent the patient to our clinic proposed a possibility of sequetrae. \#23i implant fixture was already removed 1 month ago at local clinic and antibiotics were administered for 2 weeks.

The patient had a history of skeletal class III profile with generalized poor oral health. She had undergone mandibular set-back surgery with bilateral sagittal split ramus osteotomy (BSSRO) and both paranasal augmentations using Medpor at our hospital 10 years ago. After finishing routine recall checks for orthognathic surgery, the patient had been treated with many dental implants combined with bone graft on the maxilla at another local clinic 7 years ago.

A bony like surface was exposed beneath \#22i, 23i dental prosthesis with soft tissue swelling and redness on intra-oral examination. Pus discharge was no more ob-

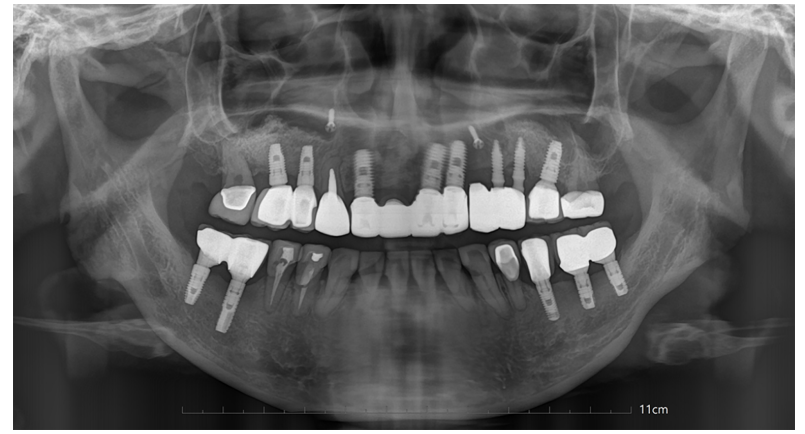

Fig. 2. Multiple dental implants were inserted on the maxilla. Especially on the left side, all teeth were replaced into dental implant except \#27. Note the mini screws near the nasal cavity on both side. It could be inferred to use for fixation of cosmetic prosthesis.

served (Fig. 1). A significant correlation of the pathology and dental origin was not observed except suspicious screws near the nasal cavity on the panoramic view (Fig. 2). These were the screws used for internal fixation of Medpore on paranasal area. In other words, the exposed structure was not an infected bone, it was the cosmetic prosthesis, Medpor.

CBCT imaging of anterior maxilla was obtained for further evaluation. The Medpor body was not identified on the CBCT images expectably, the only inference was the screws. Furthermore, bony structure was not shown on the \#23i removed site. Even alveolar bone of \#22i labial side was also not observed (Fig. 3). From this, it could be inferred that \#22i, 23i fixture was placed into the Medpor. Osseous healing might not be achieved around \#22i, 23i fixtures. \#22i fixture needed to be removed, however 4-units prosthesis connected to \#12i, 21i, 22i fixture. It was difficult to decide whether to get rid of them considering time and cost. Even the information of dental implants about the patient could not be collected because the local clinic had disappeared. After consultation with the patient, a treatment plan was established with removal of only Medpor on both paranasal area while saving the dental implant.

The operation was performed under local anesthesia with intravenous midazolam infusion for sedation. The incision was taken on the vestibule, then the infected Medpor on the left side was totally exposed. It showed discoloration as well as malodor. Once the screw loosened and removed, the Medpor was able to be detached 

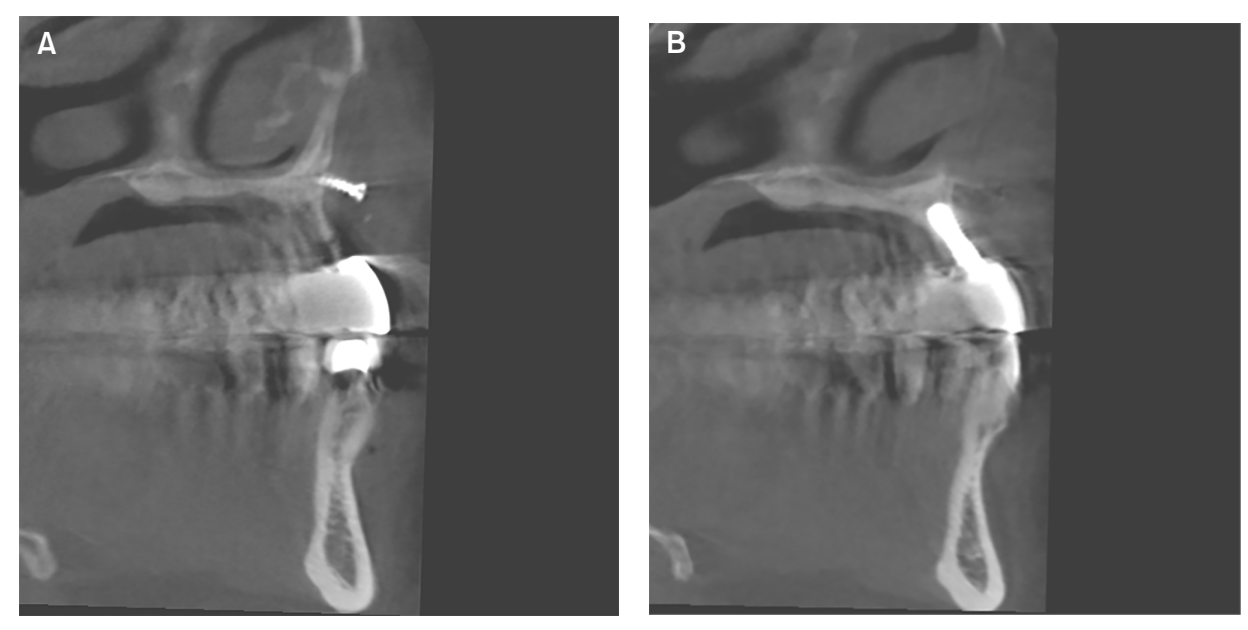

Fig. 3. CBCT imaging on the anterior maxilla. It could be inferred that peri-implant bone loss might occur under the Medpor considering the position of the screw, dental implant fixtures and Medpor. (A) This image showed the loss of alveolar bone at the \#23i extracted site and a screw under the \#23i. (B) The bone loss of \#22 labial side was observed.
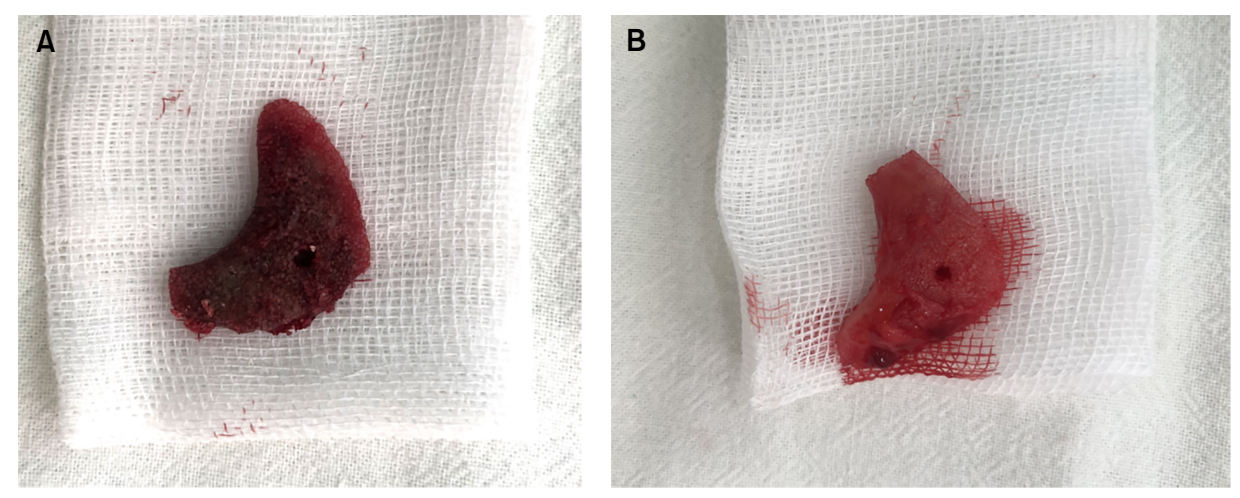

Fig. 4. Removed the paranasal Medpors from the recipient site. (A) Inner surface of the Medpor on the left was dicolorated. (B) Intact Medpor on the right was clean and well-integrated by fibrous tissue.

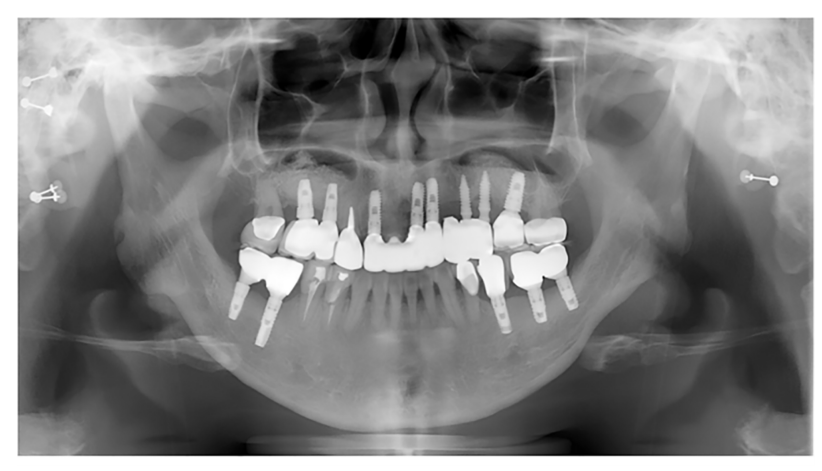

Fig. 5. Panoramic radiograph after removal of Medpors on both sides.

easily afterward, the fixture of \#22i was exposed. Meanwhile, the Medpor on the right area was clean and well-integrated into a recipient site (Fig. 4). It was not simple to remove intact Medpor due to its fibrovascular integration. In order to separate the graft, blunt dissection to all sides was performed. Primary closure was done after copious irrigation with saline. Antibiotics (Amoxicillin/Clavulanic acid 250/125 mg, three times a day),
NSAIDs and mouth rinse $(0.12 \%$ Chlorhexdine $)$ were prescribed. Stiches were removed after 7 days postoperatively. The patient's symptom was alleviated, however, the soft tissue was not closed over \#22i (Fig. 5). Recall check continued to 3 months postoperatively. Soft tissue showed migration over \#22i fixture (Fig. 6).

\section{DISCUSSION}

Cosmetic augmentation using alloplastic graft "The Medpor" has been reported with aesthetic outcome and high success rate in craniofacial surgery. It is convenient to handle during surgery. It can be contoured, trimmed, adapted and easily be fixed with screws ${ }^{11)}$. Usually, the surgical technique of paranasal augmentation with the use of Medpor is combined with BSSRO in the skeletal class III profile. It would be a good option for the patient who wants to avoid invasive surgery and improve cosmetic problems ${ }^{1,3,5}$. Medpor, the porous high-density polyethylene, has been proven stable for skeletal 

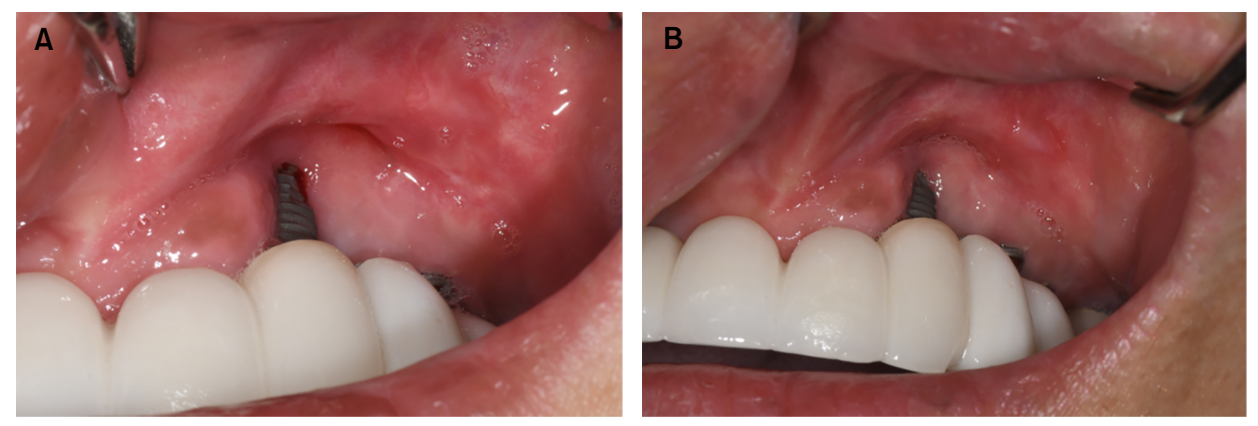

Fig. 6. Postoperative clinical photos. (A) On the 7 days postoperatively, \#23i fixture was totally exposed. (B) Soft tissue slightly covered the \#23i fixture after the 3 months postoperatively.

reconstructions. The alloplastic implant has its main advantages as it can vascularize through its pores, which permit integration on the host tissue ${ }^{4}$. However, there may be possibility of graft failure such as persistent discomfort, esthetically dissatisfaction, implant exposure and infection $^{12)}$.

The outcome of Medpor would be dependent on the recipient site. The grafts placed in the region requiring intraoral incision showed a higher risk of infection compared with other part (i.e., malar, paranasal area and mandible). The infection risk increases in oral cavity containing a high number of bacteria, ${ }^{4,11-14)}$. Unusual complications combined with paranasal Medpor and dental implant on the anterior maxilla are presented in this article. In this case, the dental implant fixtures were inserted adjacent to the Medpor body resulting in prosthesis removal. With the fact of Medpor being radiolucent, the contour is invisible on the dental radiography. Clinically, the material might mimic bony structure on the anterior maxilla. A similar hardness and texture as cancellous bone, even its white color could make an oral practitioner misinterpret.

Screw fixation of the Medpor to the facial skeleton is crucial for the graft success in order to obliterate any gaps between the Medpor and the recipient bed. The stabilization of this material should also be maintained ${ }^{4,11,13)}$. By installing dental implant near the Medpor it might be elevated, making a micro gap from the recipient site. The gaps would be a potential space for the infection ${ }^{4}$. Even mucosal sealing around dental implants of the labial side, not osseointegration, was vulnerable to infection by the formation of periodontal deep pocket. Persistent communication between oral cavity and submucosal space after dental implant prosthesis connection might be an effective route of infection.
A comprehensive examination of patient's previous history is required prior to operation. The patient is recommended to informed their history thoroughly to the medical personnel. Before placing the dental implant, preoperative diagnosis and assessment through 3-dimensional imaging should be obtained precisely on the local bone anatomy. In the anterior maxilla, it presents a significant challenge to dentist due to variable consideration, especially because of the narrow thickness of labial bone. When the dentist confronts cases similar to this article, a strategic treatment plan would be required. It would be better with a prosthetic treatment than implant placement on the anterior maxilla. A guided surgery with the CT scan would be recommended as a better option to avoid the predicted position of the cosmetic prosthesis. If there is a possibility that a dental implant inserted around the Medpor, it is important to manage the patient with routine follow up because it may not be achieved with osseous healing, which is vulnerable to peri-implantitis. Similar complications of Medpor and dental implant were not revealed on the search of the article. Therefore, this is a helpful suggestion for dental specialist to provide insight into the treatment procedures for oral and maxillofacial parts.

\section{CONCLUSION}

Medpor would be an effective material in the reconstruction of skeletal defects. For the oral and maxillofacial specialists, the paranasal area is a cautious region as being of adjacent to the dentition and mimicking the maxillary bone. When the dental implant is inserted on the anterior maxillary bone with its history, it is required special attentions as well as strategic surgical planning. In order to avoid the failure of Medpor as well 
as the dental implants, a comprehensive examination of patient history and an exact surgical plan should be obtained preoperatively. Also obtaining knowledge and having understanding of the oral and maxillofacial surgery part is important as well.

\section{INFORMED CONSENT}

Patient consent was obtained.

\section{CONFLICT OF INTEREST}

We have no conflicts of interest.

\section{REFERENCES}

1. Teriino EO. Alloplastic facial contouring: surgery of the fourth plane. Aesthetic Plast Surg 1992;16:195-212.

2. Park SB, Kim YI, Hwang DS, Lee JY. Midfacial soft-tissue changes after mandibular setback surgery with or without paranasal augmentation: cone-beam computed tomography (СBCT) volume superimposition. J Craniomaxillofac Surg 2013;41:119-23.

3. Hernández-Alfaro F, García E, Martí C, Porta A. U-shaped osteotomy in management of paranasal deficiency. Int J Oral Maxillofac Surg 2006;35:1145-8.

4. Yaremchuk MJ. Facial skeletal reconstruction using porous polyethylene implants. Plast Reconstr Surg 2003;111: 1818-27.
5. Yaremchuk MJ, Doumit G, Thomas MA. Alloplastic augmentation of the facial skeleton: an occasional adjunct or alternative to orthognathic surgery. Plast Reconstr Surg 2011;127: 2021-30.

6. Öztürk S, Sengezer M, Coskun Ü, Zor F. An unusual complication of a Medpor implant in nasal reconstruction: a case report. Aesthetic Plast Surg 2002;26:419-22.

7. Sevin K, Askar I, Saray A, Yormuk E. Exposure of high-density porous polyethylene (Medpor $\left.{ }^{\circledR}\right)$ used for contour restoration and treatment. Br J Oral Maxillofac Surg 2000;38:44-9.

8. Tolstunov L. Dental implant success-failure analysis: a concept of implant vulnerability. Implant Dent 2006;15:341-6.

9. McCarthy C, Patel RR, Wragg PF, Brook IM. Dental implants and onlay bone grafts in the anterior maxilla: analysis of clinical outcome. Int J Oral Maxillofac Implants 2003;18:238-41.

10. Sclar AG. Strategies for management of single-tooth extraction sites in aesthetic implant therapy. J Oral Maxillofac Surg 2004;62:90-105.

11. Cenzi R, Farina A, Zuccarino L, Carinci F. Clinical outcome of 285 Medpor grafts used for craniofacial reconstruction. J Craniofac Surg 2005;16:526-30.

12. de Moraes Ferreira ACR, Muñoz XMJP, Okamoto R, Pellizer EP, Garcia Jr IR. Postoperative complications in craniomaxillofacial reconstruction with Medpor. J Craniofac Surg 2016;27:425-8.

13. Niechajev I. Facial reconstruction using porous high-density polyethylene (medpor): long-term results. Aesthetic Plast Surg 2012;36:917-27.

14. Ridwan-Pramana A, Wolff J, Raziei A, Ashton-James CE, Forouzanfar T. Porous polyethylene implants in facial reconstruction: outcome and complications. J Craniomaxillofac Surg 2015;43:1330-4. 